\title{
Computer Analysis on Network Space Characteristics of Economic Connection in the Yangtze River Delta Urban Agglomeration
}

\author{
Duo Xu \\ School of economics, Shanghai University, P.R.China \\ xuduoleo@163.com
}

\begin{abstract}
Keywords: The Yangtze River Delta Urban Agglomeration; Gravity model; Economic network; Computer network analysis
\end{abstract}

\begin{abstract}
Networking development of urban agglomeration is one of essential characteristics of the integrative growth in the Yangtze River Delta region. In this paper, social network analysis (SNA) and kernel density estimation are combined through computer gravity model and structural model of urban agglomeration's economic networks is constructed. After an empirical study on 25 cities in the Yangtze River Delta, network density, kernel density distribution and cohesive subgroups of economic network in Yangtze River Delta urban agglomeration are analyzed by software UCINET and ArcGIS, to innovatively research the structure and networking development patterns of urban agglomeration. It is indicated by the results that a relatively mature net association has formed among cities in the Yangtze River Delta, while the centrality between cities in the network is imbalanced, so that relevant suggestions on network optimization of economic relation among Yangtze River Delta urban agglomeration are proposed.
\end{abstract}

\section{Research Background and Definition of Problems}

The Yangtze River Delta urban agglomeration is the largest urban agglomeration in China, as it is composed of city strip along the river and cities around Hangzhou Bay. With economic globalization and advancing urbanization, metropolitan areas and urban agglomeration network are formed. Regional economy's high-speed development enables the economic relation in urban system to be more active and develop in the unprecedented way, structure, speed and scale, gradually presenting the feature of networking.

In this paper, social network analytical method of human geography and computer kernel density method are adopted to analyze network space structure of economic relation in the Yangtze River Delta urban agglomeration; based on related data of 2014, the strength of economic relation among 25 cities in the Yangtze River Delta is measured; network density and kernel density among cities are analyzed, so as to propose feasible suggestions on the development and optimization of the Yangtze River Delta urban agglomeration.

\section{Construction of Networking Structural Model for Urban Agglomeration}

Analysis on Strength of Economic Relation. It is believed by the theory of economic gravity in economic dynamics that there is also a rule of inter-attraction in the economic relation among cities[1], as the basic gravity model is:

$$
R_{\mathrm{ij}}=k_{i j} \frac{\sqrt{P_{i} G_{i}} S_{i} \sqrt{P_{j} G_{j}} S_{j}}{D_{i j}^{2}}, k_{i j}=\frac{G_{i}}{G_{i}+G_{i}}
$$

In this formula: Rij is city $\mathrm{i}$ to city $\mathrm{j}$ economy contact strength; $\mathrm{I}, \mathrm{j}$ respectively the urban area is $\mathrm{Si}, \mathrm{Sj}$; i, j espectively city urban population of $\mathrm{Pi}, \mathrm{Pj} ; \mathrm{i}, \mathrm{j}$ respectively city GDP value of $\mathrm{Gi}, \mathrm{Gj}$; Dij is the shortest highway traffic mileage between the two cities.

The shortest highway traffic mileages between cities are adopted to be distances and parameter $\mathrm{k}$ is brought in to represent economic structural factor based on the optimal computation by Wang Xin et 
al. (2006) on urban gravity model and then correct $k_{\mathrm{ij}}$ value through the specific value of a city's GDP in the sum of two cities' GDP[2].

Analysis on Economic Network Structure of Urban Agglomeration based on Social Network Analysis. In this study, economic network structure of the Yangtze River Delta urban agglomeration is researched; as for the measurement of the structure, this study will adopts a series of quantized core indexes in the theory of social network analysis.

Integral Network Density. This index reflects the connection degree between nodes in the network, as the network density can depict the existing distribution of economic relation and degree of difference between overall complete diagram, as the basic gravity model is:

$\mathrm{D}=\sum_{i=1}^{k} \quad \sum_{j=1}^{k} \mathrm{~d}\left(n_{i}, n_{j}\right) / \mathrm{k}(\mathrm{k}-1)$

In the formula: $D$ is the network density; $K$ for the node number; $D\left(n_{i}, h_{j}\right)$ is the relationship between the node $n_{i}$ and $n_{j}$.

Analysis on Network Kernel Density. Kernel density estimation method is suitable for visual representation of point distribution pattern, as the basic gravity model is:

$\gamma \mathrm{h}(\mathrm{p})=\sum_{i=1}^{n} \frac{(1)}{h^{2}} k\left(\frac{p-p_{i}}{h}\right)$

In the formula: Gamma $h(p)$ is the density of point $p$ value; $K$ is the weighting function; (p-pi) is density value of the distance between the point $\mathrm{p}$ and $\mathrm{p}_{\mathrm{i}} ; \mathrm{H}$ is the search radius for bandwidth.

Internal Cohesive Subgroups. Cohesive subgroup is a subset of actors satisfying the following conditions and becomes more persuasive as an explanatory variable with network coherence in the sociological theory. [3]In this paper, we study the interior cohesive subgroups of urban network and the relation and interactive mode between those subgroups, so as to investigate the development status of urban agglomeration's network from the dimensionality of integral network.

\section{Empirical Analysis on Economic Relation Network in the Yangtze River Delta Urban Agglomeration}

Research Scope and Data Sources. The Yangtze River Delta Urban Agglomeration including Shanghai,Zhejiang,Jiangsu, we choose 25 cities in these province as the research object[3].According to the urban gravity constructed above, strength value of economic relations among 25 cities in the Yangtze River Delta (Fig. 1); thereafter, social network analytical tool can be adopted to conduct analysis[4], furthering the knowledge about networking structure of economic relations and providing basis for researches on its development.

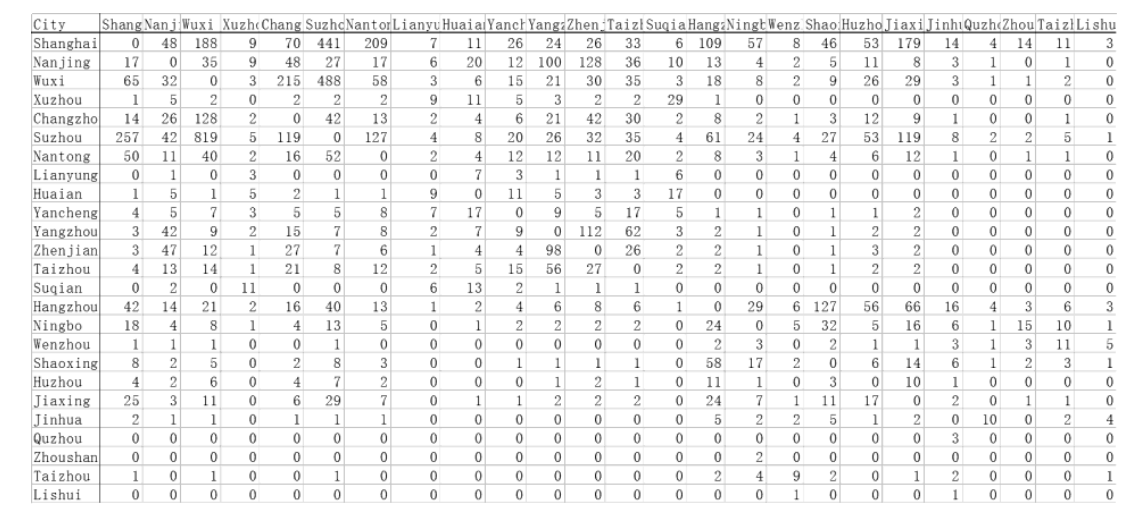

Figure. 1 Strength value of economic relations among 25 cities in the Yangtze River Delta

Visualization of Economic Network in the Yangtze River Delta Urban Agglomeration. In this paper, Ucinet software is mainly adopted to analyze the network structure of economic relation among the Yangtze River Delta urban agglomeration. [5] When the matrix data in the Fig. 2 is input 
into Ucinet Software, a binary directional topological simulation structure chart of the economic network in the agglomeration is set up[6].

As the Fig. 2 reflects, the critical value of relation strength set in the topological simulation chart of Yangtze River Delta urban agglomeration's economic network is 0 . The arrow lines in the figure means the economic relation's influence from one city to another.

It can be known from the Fig. 2 that, Shanghai, Suzhou and Hangzhou are of remarkably higher centrality in the center of network, meaning the three cities occupies the important central status in this economic relation network[7]; meanwhile, Wuxi, Ningbo, Nanjing, Nantong and Shaoxing also play the role of communication in the network.

We can see that there are disparities of economic links bjmetween cities in the Yangtze River Delta city group through the directed arrows[8],for example, there are strong economic links between Shanghai, Suzhou, Wuxi, Hangzhou, Nanjing. The mutual influence and the development of synergy between the non coastal cities in north and south of Yangtze River Delta city group is yet to be strengthened.

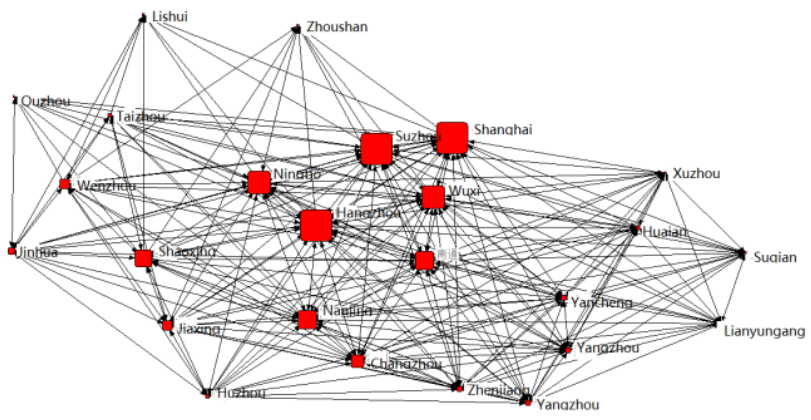

Figure. 2 The topology of the network structure of economic relation among the Yangtze River Delta urban

Research on Network Structure in the Yangtze River Delta Urban Agglomeration. Analysis on Network Density of Urban Agglomeration

Analysis on Network Density of Urban Agglomeration. Network density is calculated by means of Ucinet Software[9], and the result shows that the network density value of the Yangtze River Delta urban agglomeration at present is 0.6550 , showing relatively high density of the economic density and strong economic relation. However, that the network density is larger isn't better, since the transaction costs and expenses for building economic relation among cities will influence the utilization efficiency of resources instead.

Analysis on Network Kernel density of Economic Relation in the Yangtze River Delta Urban Agglomeration. The ArcGIS software is used to analyze the corresponding nuclear density, and we got the density distribution map of the economic link intensity of the Yangtze River Delta city group (Fig. 3).From the perspective of the whole Yangtze River Delta urban agglomeration, the kernel density distribution of the integral economic relation is centered on Suzhou, Wuxi and Changzhou, gradually declining towards the outer circle layer. Shanghai, Suzhou, Wuxi and etc., as representatives of central city in the urban agglomeration, show the kernel characteristics of higher density[10]; as a result, the aggregation area of economic relation network in the Yangtze River Delta and the trend of economic relation can be known by kernel density analysis. It can be seen that the economic relation of the urban agglomeration is quite centralized on the whole, while the detailed economy can't be totally reflected; as a result, the economic relation status of network subgroups has to be refined further. 


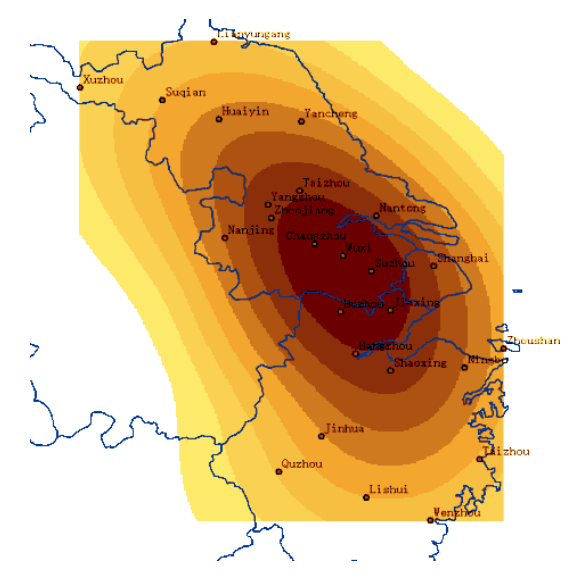

Figure. 3 The kernel density distribution of the integral economic relation of Yangtze River Delta urban agglomeration

Analysis on Network's Cohesive Subgroups in Urban Agglomeration. The analysis on cohesive subgroups is based on the strength of economic relation among cities, to discuss the aggregation phenomenon of cities in the Yangtze River Delta urban agglomeration[11]. In this study, concor algorithm in Unicet Software is adopted to acquire the specific composition of all cohesive subgroups, which is which is shown in the Fig. 4.

1) Ten cities, including southeast part of Jiangsu and northeast Zhejiang, form relatively tighter economic relations with Shanghai as the center, posing economic radiation of different degrees on other 5 cohesive subgroups, so that is can be believed that the network development of the Yangtze River Delta urban agglomeration is based on this cohesive subgroup.

2) Jiangsu has 13 prefecture-level cities, while the economic relations among them are imbalanced and the central city hasn't exerted it radiation and spreading effects. Cities in south Jiangsu have frequent economic activities with Shanghai; Nanjing links up the economic relation between Shanghai and periphery districts of Yangtze River Delta.

3) It can be know from the structure chart of Zhejiang's cohesive subgroups, the economic relations among urban agglomerations of Zhejiang are relatively weaker, and Hangzhou and Ningbo act as the important parts connecting the economic activities of Shanghai with other urban agglomeration in Zhejiang.

In the integration process of the Yangtze River Delta, urban agglomeration presents a net-structure multi-dimensional nesting mode and some urban subgroups around a certain center can easily be isomorphic on the economic structure and activity relationship. All city subjects have to strength further the regional communication and cross-border cooperation, gradually forming a multicenter and networking development pattern.

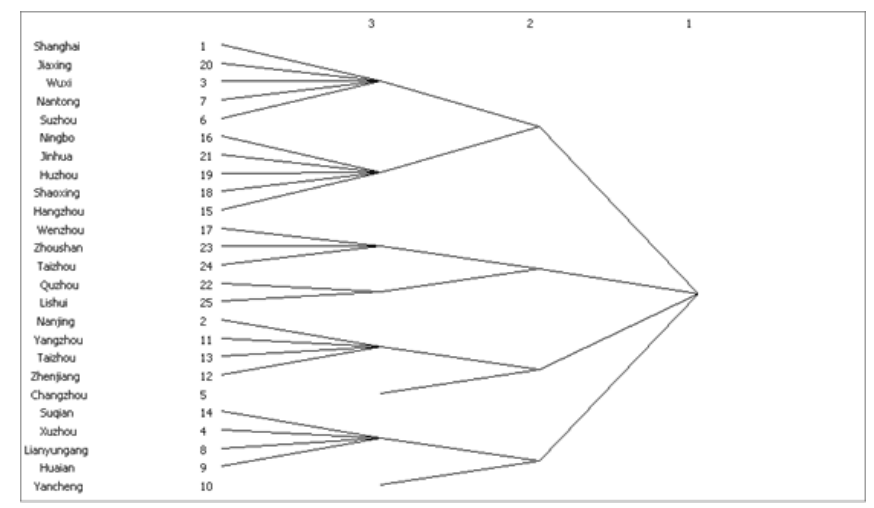

Figure. 4 Cohesive subgroups of Yangtze River Delta urban agglomeration 


\section{Conclusion and Relevant Policy Suggestions}

In this paper, the economic relation network of two provinces and one city in the Yangtze River Delta urban agglomeration, that is, 25 cities in total, is taken as an example; with the social network analytical tool, the urban agglomeration is analyzed quantitatively and qualitatively, reaching several conclusions shown as below:

1) the overall network density of the Yangtze River Delta urban agglomeration is at a higher level, economic relation among cities are quite tight on the whole, while the centrality is obviously imbalanced;

2) the development of the Yangtze River Delta urban agglomeration is benefited from the radiation and drive of core cities like Shanghai, Suzhou and Hangzhou to their surrounding cities;

3) other cities speed up the pace to connect Shanghai from all sides and fully receive comprehensive service from Shanghai.

According to the empirical research on network structure of urban agglomeration, the relation between all city nodes and overall network can be clearly seen, and related policy suggestions are also provided.

1)Enhance the correlation degree among cities in the Yangtze River Delta and develop network cooperation function and effect. Speed up the urban integration process of the area and effectively enhance resource allocation and production and trading efficiency among cities.

2)Optimize the internal networking hierarchical structure of urban agglomeration, encourage the cities in the Yangtze River Delta to actively construct multilateral relations crosswise, integrate the scattered resource factors across boundary in the network. Small- and medium-scale cities should continue to reinforce their overall network relation to achieve diversified competitions.

3)Establish a networking governance mechanism for the Yangtze River Delta urban agglomeration and set an integrative goal for the regional development through innovation of regional integration policies and systems, to build up a benefit-sharing and cooperative network governance mechanism for the urban agglomeration with trust and mutual benefits.

\section{References}

[1] Meng Deyou,Lu Yulin. Strength and Direction of Regional Economic Linkage in Jiangsu Province Based on Gravity Model. Progress in Geography,2009,28(5), p697 703

[2] Wang Xin, Wu Dianting, Wang Hongqiang. An attempt to calculate economic links between cities. Urban Studies, 2006(3),p55-59

[3] Luo Jiade. Social network analysis. Beijing Social Science Literature Press, 2005.

[4] Tang Fanghua, Tang Hui, Sun Qian. Analysis of the economic network structure of urban agglomerations in the middle Yangtze River. Acta Geographica Sinica， 2013(10),p1357 1366

[5] Li Guoping, Wang Liming,Yang Kaizhong. The measurement and analysis of economic relationship between shenzhen and zhujiang delta. Economic Geography,2001,21(1),p33 37

[6] Xiong Jianping, Liu Chengliang, Yuan Jun. Progressin foreign research on economic linkages of city agglomeration. World Regional Studies, 2006( 1),p63 70

[7] Wang Jue, Chen Wen, Yuan Feng. Human mobility and evolution based on social network:An empirical analysis of Yangtze River Delta.Geographical Research,2014(2),p385 400

[8] Xiang Yunbo, Peng Xiufen, Xu Changle. Research on economic linkage between shanghai and changjiang economic zone. Resources and Environment in the Yangtze Basin,2009(6),p508 514

[9] Friedmann J R.The world city hypothesis.Development and Change,1986,17( 1),p59 83 
[10]Taylor P J, Hoyler M.The spatial order of European cities under conditions of contemporary globalizati. Tijdschrift voor Economische en Sociale Geografie,2000( 2), p176 189

[11]Meijers E. Polycentric Urban Regions and the Quest for Synergy:Is a Network of Cities More than the Sum of the P arts?.Urban Studies,2005,42( 5),p $765 \sim 781$ 